IFF-5/92

May 1992

\title{
On Integrable Quantum Group Invariant
}

\author{
Antiferromagnets* \\ R. Cuerno ${ }^{\dagger}$ G. Sierra ${ }^{\ddagger}$ \\ Instituto de Matemáticas y Física Fundamental, CSIC \\ Serrano 123, E-28006 Madrid, SPAIN \\ C. Gómez ${ }^{\S}$ \\ Départment de Physique Théorique \\ Université de Genéve, CH-1211 Geneva, SWITZERLAND
}

\begin{abstract}
A new open spin chain hamiltonian is introduced. It is both integrable (Sklyanin's type $K$ matrices are used to achieve this) and invariant under $\mathcal{U}_{\epsilon}(s l(2))$ transformations in nilpotent irreps for $\epsilon^{3}=1$. Some considerations on the centralizer of nilpotent representations and its representation theory are also presented.
\end{abstract}

${ }^{*}$ Lecture given by C.G. at the Winter School on Theoretical Physics, Wroclaw, Poland, February 1992. Keywords: Integrable models. Open chains. Quantum groups. 1991 MSC: 82B23, 17B37. PACS: 05.50.+q, 02.90.+p, 75.10 Jm

$\dagger^{\dagger}$--mail: IMTRC59@CC.CSIC.ES. Supported by a PFPI grant of the spanish MEC.

${ }^{\ddagger} \mathrm{e}-$ mail: SIERRA@CC.CSIC.ES

§e-mail: GOMEZC@SC2A.UNIGE.CH. Permanent address: IMFF, CSIC. 
The most direct way to get into the physics associated with quantum groups is certainly the study of quantum mechanical systems possesing a quantum group symmetry. Some examples of this kind are already known in the context of one dimensional spin chains [1]. The simplest one is the XXZ spin-1/2 chain with boundary conditions:

$$
H=\sum_{i=1}^{N-1} \sigma_{i}^{x} \sigma_{i+1}^{x}+\sigma_{i}^{y} \sigma_{i+1}^{y}+\frac{q+q^{-1}}{2} \sigma_{i}^{z} \sigma_{i+1}^{z}+\frac{q-q^{-1}}{2}\left(\sigma_{1}^{z}-\sigma_{N}^{z}\right)
$$

which is invariant under $\mathcal{U}_{q}(s l(2))$ transformations. The integrable version of the spin one Heisenberg model with non vanishing anisotropy is the ZamolodchikovFateev hamiltonian [2]:

$$
\begin{aligned}
H^{Z F}= & \sum_{i=1}^{N-1} H_{i, i+1}^{Z F} \\
= & \sum_{i=1}^{N-1} S_{i}^{x} S_{i+1}^{x}+S_{i}^{y} S_{i+1}^{y}+\frac{q^{2}+q^{-2}}{2} S_{i}^{z} S_{i+1}^{z}-\left(S_{i}^{x} S_{i+1}^{x}+S_{i}^{y} S_{i+1}^{y}\right)^{2} \\
& -\frac{q^{2}+q^{-2}}{2}\left(S_{i}^{z} S_{i+1}^{z}\right)^{2}+\left(1-q-q^{-1}\right)\left[\left(S_{i}^{x} S_{i+1}^{x}+S_{i}^{y} S_{i+1}^{y}\right) S_{i}^{z} S_{i+1}^{z}+\leftrightarrow\right] \\
& +\left(\frac{q^{2}+q^{-2}}{2}-1\right)\left(\left(S_{i}^{z}\right)^{2}+\left(S_{i+1}^{z}\right)^{2}\right)
\end{aligned}
$$

The hamiltonian $H_{i, i+1}^{Z F}$ is given by the logarithmic derivative of the spin one $R$-matrix $R^{(s=1)}(u)$ of the affine Hopf algebra $\mathcal{U}_{q}(\widehat{s l(2)})$. In order to make the ZF hamiltonian invariant under global $\mathcal{U}_{q}(s l(2))$ transformations the following boundary term should be added

$$
H^{(B)}=\frac{q^{2}+q^{-2}}{2}\left(S_{N}^{z}-S_{1}^{z}\right)
$$

Integrability in a box requires that the Sklyanin reflection operators $K_{ \pm}(u)$, which describe the scattering with the wall obey the relations [3]:

$$
R_{12}(u-v) \stackrel{1}{K}-(u) R_{12}(u+v) \stackrel{2}{K}-(v)=\stackrel{2}{K}-(u) R_{12}(u+v) \stackrel{1}{K}-(u) R_{12}(u-v)
$$


For the quantum group invariant hamiltonian $H^{Z F}+H^{B}$ it is not hard to prove [1] that:

$$
\left[H^{Z F}+H^{B}, t(u)\right]=0
$$

where the box transfer matrix $t(u)$ is defined by:

$$
t(u)=\operatorname{Tr}\left(K_{+}(u) T(u) K_{-}(u) T^{-1}(-u)\right)
$$

with $K_{-}$satisfying (勾) for $R$ the spin one $R$-matrix of $\mathcal{U}_{q}(\widehat{s l(2)})$ and $K_{+}(u)=$ $K_{-}(-u-\eta), q=\exp \eta$. From (5) it follows the integrability of the hamiltonian $H^{Z F}+H^{B}$. The monodromy matrix $T(u)$ in (6) is the one defined by the $s=1$ quantum $R$ matrix of $\mathcal{U}_{q}(\widehat{s l(2)})$.

In reference [5] a one parameter family of integrable deformations of (2) was defined for $q=\epsilon, \epsilon^{3}=1$ :

$$
\begin{aligned}
& H(\lambda)= 2\left(\epsilon-\epsilon^{-1}\right) \sum_{i=1}^{N} \frac{\lambda \epsilon+\lambda^{-1} \epsilon^{-1}}{2}\left(S_{i}^{x} S_{i+1}^{x}+S_{i}^{y} S_{i+1}^{y}\right) \\
&-\frac{1}{2} S_{i}^{z} S_{i+1}^{z}-\left(S_{i}^{x} S_{i+1}^{x}+S_{i}^{y} S_{i+1}^{y}\right)^{2}+\frac{1}{2}\left(S_{i}^{z} S_{i+1}^{z}\right)^{2} \\
&+\left(\frac{\lambda \epsilon+\lambda^{-1} \epsilon^{-1}}{2}+\omega\right)\left[\left(S_{i}^{x} S_{i+1}^{x}+S_{i}^{y} S_{i+1}^{y}\right) S_{i}^{z} S_{i+1}^{z}+\leftrightarrow\right]-\frac{3}{2}\left(\left(S_{i}^{z}\right)^{2}+\left(S_{i+1}^{z}\right)^{2}\right) \\
&-\frac{\lambda \epsilon-\lambda^{-1} \epsilon^{-1}}{2\left(\epsilon-\epsilon^{-1}\right)}\left(S_{i}^{x} S_{i+1}^{x}+S_{i}^{y} S_{i+1}^{y}\right)\left(S_{i}^{z}+S_{i+1}^{z}\right)+\frac{\lambda^{2} \epsilon^{-1}-\lambda^{-2} \epsilon}{2\left(\epsilon-\epsilon^{-1}\right)}\left(S_{i}^{z}+S_{i+1}^{z}\right) \\
& \equiv \sum_{i=1}^{N} H(\lambda)_{i, i+1} \\
& \omega(\lambda)=\sqrt{\left(\lambda-\lambda^{-1}\right)\left(\lambda \epsilon^{-1}-\lambda^{-1} \epsilon\right)}
\end{aligned}
$$

The hamiltonian $H(\lambda)_{i, i+1}$ is (up to a constant factor) the logarithmic derivative of the quantum $R$-matrix $R^{(\lambda)}(u)$ which intertwines nilpotent representations of $\mathcal{U}_{q}(\widehat{s l(2)})$ for $q=\epsilon$ 河, 同. Let us recall that nilpotent representations of $\mathcal{U}_{\epsilon}(\widehat{\operatorname{sl}(2)})$ $\left(\epsilon^{3}=1\right)$ are three-dimensional irreducible representations transforming under 
the central Hopf subalgebra as: $E^{3}=F^{3}=0, K^{3}=\lambda^{3}$, with $\lambda$ a generic complex number. For $\lambda=\epsilon^{2}$, which corresponds to the spin one representation, $H(\lambda)$ coincides with $2\left(\epsilon-\epsilon^{-1}\right) H^{Z F}$ for $q=\epsilon$.

The problem we want to address in this lecture is the definition of an integrable and quantum group invariant version of (7). Quantum group invariance is easily obtained adding to (7) the boundary term:

$$
H^{B}(\lambda)=\omega^{2}\left(S_{1}^{z}-S_{N}^{z}\right)
$$

The hamiltonian $H(\lambda)+H^{B}(\lambda)$ coincides, for $\lambda=\epsilon^{2}$, with $2\left(\epsilon-\epsilon^{-1}\right)\left(H^{Z F}+H^{B}\right)$, which is already a good indication concerning integrability. However, to attain it, we need to check for $H(\lambda)+H^{B}(\lambda)$ the equivalent to equation (5) with $K$ now being a solution to (4) for $R$ the quantum nilpotent $R^{\lambda}(u)$-matrix. The $K$-matrices for the nilpotent $R^{\lambda}(u)$-matrix are:

$$
\begin{aligned}
K_{-}(u)= & \frac{1}{\sinh \alpha_{-} \sinh \left(\alpha_{-}-\eta\right)} \\
\times & \operatorname{diag}\left(\sinh \left(u+\alpha_{-}\right) \sinh \left(u+\alpha_{-}-\eta\right),-\sinh \left(u-\alpha_{-}\right) \sinh \left(u+\alpha_{-} \eta\right),\right. \\
& \left.\sinh \left(u-\alpha_{-}\right) \sinh \left(u-\alpha_{-}+\eta\right)\right) \\
K_{+}(u)= & \operatorname{diag}\left(\sinh \left(u+\eta-\alpha_{+}\right) \sinh \left(u-\alpha_{+}-\eta\right),\right. \\
& \left.-\sinh \left(u+\eta+\alpha_{+}\right) \sinh \left(u-\alpha_{+}-\eta\right), \sinh \left(u+\alpha_{+}\right) \sinh \left(u+\alpha_{+}+\eta\right)\right)
\end{aligned}
$$

with $\alpha_{ \pm}$free parameters and $\eta=2 \pi \mathrm{i} / 3$. Note that these matrices possess precisely the same form as those used in reference [4] to prove the integrability of the Zamolodchikov-Fateev spin one chain with boundary terms. Using these $K$ matrices we derive for $H(\lambda)+H^{B}(\lambda)$ the integrability condition (5) by showing that $H(\lambda)+H^{B}(\lambda)$ is proportional to the second derivative, at the point $u=0$, of the box transfer matrix $t(u)$, for $\alpha_{ \pm}=\infty$. Notice the diference with the ZF case where the hamiltonian is given by the first logarithmic derivative of $t(u)$. 
The reasons are that in our case, as it can be seen from (9), $t(0)=\operatorname{Tr} K_{+}(0)$ becomes zero, and that $\operatorname{Tr}\left(\stackrel{0}{K}_{+}(0) H(\lambda)_{N 0}\right) \propto \stackrel{N}{\mathbf{1}}$.

The nice thing about a quantum group invariant hamiltonian is that most of the properties of the spectrum can be directly derived from representation theory. So for instance, for the hamiltonian (1) we know that each energy eigenvalue is associated with a given spin-j irrep of $\mathcal{U}_{q}(s l(2))$ and that it would be $(2 j+1)$ times degenerate. The different irreps that can appear in the spectrum are the ones obtained by decomposing $\stackrel{N}{\otimes} V^{1 / 2}$. Moreover the different hwv's transforming in the same representation $\mathrm{j}$ will define irreducible representations of the centralizer of $\mathcal{U}_{q}(s l(2))$, which for spin $1 / 2$ is given by the Hecke algebra. In the massless phase $(|q|=1)$, the previous results provide, together with the systematic use of the finite size technology [7], the basis for the quantum group interpretation of conformal field theories [8]. A similar study can now be done for the hamiltonian $H(\lambda)+H^{B}(\lambda)$ with the new features being associated to the peculiarities of the representation theory at roots of unit.

In what follows we will concentrate our analysis on the structure of the centralizer for nilpotent representations of $\mathcal{U}_{\epsilon}(\operatorname{sl}(2))$. Given a nilpotent representation $V^{\lambda}$ we define the centralizer $C_{N}^{\lambda}(\epsilon)$ as the algebra of endomorphisms $g: \stackrel{N}{\otimes} V^{\lambda} \rightarrow \stackrel{N}{\otimes} V^{\lambda}$ commuting with the quantum group action. To get the generators of $C_{N}^{\lambda}(\epsilon)$ we first define the "braiding limit" of the quantum $R$-matrix $R^{\lambda \lambda}(u)$ of the affine Hopf algebra $\mathcal{U}_{\epsilon}(\widehat{s l(2)})$ as follows:

$$
R_{ \pm}^{\lambda}=\lim _{u \rightarrow \pm \infty} R^{(\lambda, \lambda)}(u)_{r_{1} r_{2}}^{r_{1}^{\prime} r_{2}^{\prime}} e^{u\left(r_{1}-r_{2}^{\prime}\right)}
$$

Elements in $C_{N}^{\lambda}(\epsilon)$ are then generated by

$$
g_{i}^{ \pm}=\mathbf{1} \otimes \cdots \otimes\left(R_{ \pm}^{\lambda}\right)_{i, i+1} \otimes \cdots \otimes \mathbf{1} \quad i=1, \ldots, N-1
$$

Based on the spectral decomposition of $R_{ \pm}^{\lambda}$ we will assume that the set of 
generators $g_{i}$ is complete. In order to get some insight into the structure of the centralizer we will first consider the case $\epsilon^{4}=1$. In this case the nilpotent representations are two-dimensional and the "braiding limit" $R$ matrix is given by:

$$
\begin{aligned}
R^{\lambda} & =\left(\begin{array}{cccc}
\lambda & & & \\
\lambda-\lambda^{-1} & 1 & \\
1 & 0 & \\
& & -\lambda^{-1}
\end{array}\right) \\
& =\sigma^{+} \otimes \sigma^{-}+\sigma^{-} \otimes \sigma^{+}+\frac{1}{2} \lambda^{-1}\left(\sigma^{z} \otimes \mathbf{1}\right)+\frac{1}{2} \lambda\left(\mathbf{1} \otimes \sigma^{z}\right)+\frac{\lambda-\lambda^{-1}}{2} \mathbf{1} \otimes \mathbf{1}
\end{aligned}
$$

This $R$ matrix has two eigenvalues, $\lambda$ and $-\lambda^{-1}$. The generators $g_{i}$ satisfy the Hecke relation:

$$
g_{i}^{2}=\left(\lambda-\lambda^{-1}\right) g_{i}+\mathbf{1}
$$

This means that the centralizer $C_{N}^{\lambda}(\epsilon)$ in the case where $\epsilon=e^{\pi \mathrm{i} / 2}$ gives us a representation of the Hecke algebra $H_{N}\left(\lambda^{2}\right)$. It is well known that for generic $q$ the irreducible representations of $H_{N}(q)$ are in one to one correspondence with irreps of $S_{N}$, see figure 1. So we may ask which representations we get from the centralizer $C_{N}^{\lambda}(\epsilon)$. At this point it is worthwhile to recall that the centralizer $C_{N}^{1 / 2}(q)$ for the spin $1 / 2$ representation of $\mathcal{U}_{q}(s l(2))$ is the quotient of a Hecke algebra $H_{N}(q)$ by the relation

$$
g_{i} g_{i+1} g_{i}+g_{i} g_{i+1}+g_{i+1} g_{i}+g_{i}+g_{i+1}+1=0
$$

which in turn is equivalent to reducing the allowed Young tableaux to those with at most two rows.

In this case the $R^{1 / 2}$ matrix which intertwines two spin $1 / 2$ irreducible rep- 


\section{Figure 1:}

resentations of $\mathcal{U}_{q}(s l(2))$ is given by:

$$
R^{1 / 2}=\left(\begin{array}{cccc}
q & & & \\
& 0 & q^{1 / 2} & \\
& q^{1 / 2} & q-1 & \\
& & & q
\end{array}\right)
$$

This $R$-matrix has also two eigenvalues, -1 and $q$, but the main difference with respect to the $R$-matrix (12) is that in this case the multiplicities of the eigenvalues are 2 and 2, while for (12) they were 1 and 3 . The latter fact can be understood from the decomposition rule $\frac{1}{2} \otimes \frac{1}{2}=0 \oplus 1$ (irrep 0 has dimension 1 and irrep 1 is three-dimensional). More generally we see that condition (14) imposes a one to one relation between the irreps of the centralizer $C_{N}^{1 / 2}(q)$ and the decomposition into irreps of $\mathcal{U}_{q}(s l(2))$ of $\stackrel{N}{\bigotimes}^{1 / 2}$. All this means that the Brauer-Weyl theory also applies to the spin $1 / 2$ representation of $\mathcal{U}_{q}(\operatorname{sl}(2))$.

For the centralizer $C_{N}^{\lambda}(\epsilon)$ we now try to follow the same steps, namely to see which are the allowed Young diagrams in figure 1 according to the decomposition rules of nilpotent irreps. It was shown in [9, 10] that the decomposition rules of 
nilpotent irreps for generic values of $\lambda$ are given by:

$$
\lambda \otimes \lambda=\bigoplus_{i=0}^{N^{\prime}-1} \lambda^{2} \epsilon^{-2 i}
$$

where $\epsilon^{N}=1\left(N^{\prime}=N\right.$ for $N$ odd and $N^{\prime}=N / 2$ for $N$ even). In the case of $\epsilon=e^{\mathrm{i} \pi / 2}$ equation (16) explains the multiplicities $(2,2)$ of the eigenvalues of the $R^{\lambda}$ matrix (12), since $\lambda \otimes \lambda=\lambda^{2} \oplus\left(-\lambda^{2}\right)$ and both $\lambda^{2}$ and $-\lambda^{2}$ have dimension 2. Moreover the generators $g_{i}$ constructed out from $R^{\lambda}$ satisfy instead of the relation (14) the following one:

$$
\begin{aligned}
e_{i}^{-} e_{i+2}^{-} e_{i+1}^{+} e_{i}^{+} e_{i+2}^{+} & =e_{i}^{-} e_{i+2}^{-} e_{i+1}^{-} e_{i}^{+} e_{i+2}^{+}=0 \\
e_{i}^{ \pm} & \equiv \frac{\mathbf{1} \pm \lambda^{ \pm 1} R^{\lambda}}{1+\lambda^{ \pm 2}}
\end{aligned}
$$

which implies that the allowed Young diagrams, in the nilpotent case, are those of "corner" type:

The Bratelli diagram describing the centralizer $C_{N}^{\lambda}\left(\epsilon=e^{\mathrm{i} \pi / 2}\right)$ is that in figure 2. We notice that the Young diagrams of the type (18) are precisely the only ones that contribute to the Alexander-Conway polynomial as shown by Jones in [11. We would like also to mention that the $R$-matrix (12) coincides with the intertwiner $R$ matrix for the fundamental representation of $\mathcal{U}_{q}(s l(1,1)$ ) (with $q$ replaced by $\lambda$ ), which was used in reference 12] in order to construct the Alexander polynomial. It has also been found in 13 in the context of boson 
Figure 2:

Figure 3:

representations of $\mathcal{U}_{q}(s l(2))$. All this seems to indicate alternative descriptions of the nilpotent irreps of $\mathcal{U}_{\epsilon}(s l(2))$ for $\epsilon=e^{\mathrm{i} \pi / 2}$.

Coming back to our problem, we can now compare the Bratelli in figure 2 with the one we derive from the decomposition rule (16) in the case of $\epsilon=e^{\mathrm{i} \pi / 2}$, shown in figure 3 . It is then clear that the diagrams in figures 2 and 3 can be related under some identifications, as in figure 4. We now face two posibilities, either

- the set of generators $g_{i}$ given by (11) is not complet in the sense that the centralizer $C_{N}^{\lambda}(\epsilon)$ is bigger, or

- the centralizer $C_{N}^{\lambda}(\epsilon)$ is nothing but the one generated by the $g_{i}$ 's with Bratelli given by that in figure 2 and then the Brauer-Weyl theory is not working in the standard way for nilpotent representations. 
Figure 4:

Figure 5:

We believe that the correct possibility is the last one and we shall present computational evidence for this.

We shall consider the next non-trivial case, $\epsilon^{3}=1$; the Bratelli diagram for the centralizer is given in figure 5. Let us compare for instance level 3 of figure 5 with the decomposition $V^{\lambda} \otimes V^{\lambda} \otimes V^{\lambda}$ depicted in figure 6. The basis of $V^{\lambda}$ is $\left\{e_{i}\right\}_{i=0}^{2}$ and $M\left(e_{r_{1}} \otimes e_{r_{2}} \otimes e_{r_{3}}\right)=r_{1}+r_{2}+r_{3}$. In the figure each dot stands for one of the linearly independent states for each value of $M$. Dots linked by vertical lines are connected by the action of the quantum group generators on the space $V^{\lambda} \otimes V^{\lambda} \otimes V^{\lambda}$, and so they have the same energy eigenvalue. They also share the same eigenvalue of the quantum group generator $\Delta^{(3)}(K)$, which is also given in the figure. We realize that the different irreps appearing in figure 


\section{Figure 6:}

5 are one to one related with sets of irreps in figure 6 possessing the same value of $M$. In fact it can be explicitly checked that the "braiding" transformations $g_{i}$ defined by (11) close in the subspace defined by the same value of $M$. From this we can conclude that if $C_{N}^{\lambda}(\epsilon)$ is generated by the $g_{i}$ 's then Brauer-Weyl theory can not be directly applied to the case of nilpotent irreps. Certainly this result doesn't rule out the possibility of additional generators; however the explicit analysis of the spectrum of the hamiltonian $H(\lambda)+H^{B}(\lambda)$, presented in the first part of this lecture, seems to indicate that this is not the case.

For $\epsilon^{3}=1$ and a chain of 3 sites the dependences on $\lambda$ of the energy eigenvalues $E_{1}, \ldots, E_{9}$ (see figure 6) of $H(\lambda)+H^{B}(\lambda)$ are given in figure 7 . By direct inspection of this figure we see that the energy eigenvalues correponding to the same eigenvalue of $M$ have a similar behaviour. It is worth mentioning that the Bratelli diagrams in figures 2 and 5 can be derived from a modification of the decomposition rule (16). Indeed if we supplement the irrep $\lambda$ with a new 
Figure 7:

quantum number $n \in \mathbf{N}$, and considering the fusion rule

$$
\left(\lambda_{1}, n_{1}\right) \otimes\left(\lambda_{2}, n_{2}\right)=\bigoplus_{r=0}^{N^{\prime}-1}\left(\lambda_{1} \lambda_{2} \epsilon^{-2 r}, n_{1}+n_{2}+r\right)
$$

we then obtain for $N^{\prime}=2$ and 3 the Bratellis of figures 2 and 5 respectively.

This new quantum number $n$ is quite likely the Casimir of an algebra whose representations are identical to the nilpotent irreps of $\mathcal{U}_{\epsilon}(\operatorname{sl}(2))$. This is indeed the case of $N^{\prime}=2$ and $\infty$, where this algebra is $\mathcal{U}_{q}(g l(1,1))$ [14 and $\mathcal{U}\left(h_{4}\right)$ [15] respectively.

Summarizing the content of this lecture:

1. We have obtained an integrable quantum group invariant spin chain hamiltonian for nilpotent representations of $\mathcal{U}_{q}(s l(2))$ at roots of unit.

2. We have defined the centralizer for nilpotent representations $C_{N}^{\lambda}(\epsilon)$ and studied its representation theory. It turns out that the irreps of the centralizer generated by the $g_{i}$ 's in equation (11) are one to one related (in the case $\left.\epsilon^{4}=1\right)$ to irreps of $S_{N}$ characterized by "corner" type Young 
diagrams.

Many questions remain open. Among them it would be interesting to provide a proof that $C_{N}^{\lambda}(\epsilon)$ is in fact generated by the "braiding" limit (10) of the quantum $R$-matrix $R^{\lambda \lambda}(u)$, and generalize to this case the Brauer-Weyl theory. From a more speculative point of view the situation concerning the centralizer we are facing here strongly recalls the existence in CFT of extensions of chiral algebras 16].

A more detalied presentation of the content of this lecture is at present in preparation [17.

\section{References}

[1] V. Pasquier and H. Saleur, Nucl. Phys. B330 (1990), 523.

[2] A. B. Zamolodchikov and V. A. Fateev, Sov. Jour. Nucl. Phys. 32 (1980), 298.

[3] E. K. Sklyanin, J. Phys. A: Math. Gen. 21 (1988), 2375.

[4] L. Mezincescu, R. I. Nepomechie and V. Rittenberg, Phys. Lett. A147 (1990), 70 .

[5] C. Gómez and G. Sierra, "New Integrable Deformations of Higher Spin Heisenberg-Ising Chains", preprint IFF-2/92.

[6] A. Berkovich, C. Gómez and G. Sierra, "On a new class of integrable models", to appear in Intern. Jour. Mod. Phys. A.

[7] H. W. Blöte, J. L. Cardy and M. P. Nightingale, Phys. Rev. Lett. 56 (1986), 742 . 
I. Affleck, Phys. Rev. Lett. 56 (1986), 746.

J. L. Cardy, J. Phys. A: Math. Gen. 17 (1984), L385.

J. L. Cardy, Nucl. Phys. B270 [FS16] (1986), 186.

[8] A. A. Belavin, A. M. Polyakov and A. B. Zamolodchikov, Nucl. Phys. B241 (1984), 333 .

[9] C. Gómez, M. Ruiz-Altaba and G. Sierra, Phys. Lett. B265 (1991), 95.

C. Gómez and G. Sierra, Nucl. Phys. B373 (1992), 761.

[10] D. Arnaudon, Ecole Polytechnique preprint, 1991

[11] V. F. R. Jones, Ann. Math. 126 (1987), 335.

[12] L. H. Kauffman and H. Saleur, Commun. Math. Phys. 141 (1991), 293.

[13] M. L. Ge, X. F. Liu and C. P. Sun, Lett. Math. Phys. 23 (1991), 169.

[14] L. Rozansky and H. Saleur, Nucl. Phys. B to appear.

[15] C. Gómez and G. Sierra, "Quantum Harmonic Oscillator Algebra and Link Invariants", preprint October 1991.

[16] G. Moore and N. Seiberg, "Lectures on RCFT", in "Physics, Geometry and Topology", ed. by H. C. Lee, NATO ASI Series, vol. 238, Plenum 1990, and references therein.

[17] R. Cuerno, C. Gómez and G. Sierra, in preparation. 